\title{
Right Knee Chronic Prosthetic Arthritis Due to Tropheryma whippelii: A Case Report
}

\section{Díaz-Cañestro $\mathbf{M}^{1 *} \mid$ Camprodón $\mathbf{A}^{3}$ | Vidal-Lampurdanes $\mathbf{M}^{2} \mid$ Ramos-Asensio $\mathbf{R}^{4} \mid$ Riera $\mathbf{M}^{1} \mid$ Murillas J ${ }^{1}$}

*Correspondence: Díaz-Cañestro M

Address: ${ }^{1}$ Servicio de Medicina Interna-Infecciosas, Hospital Universitari Son Espases, Instituto de Investigación Sanitaria Illes Balears (IdISBa), Palma de Mallorca, Spain; ${ }^{2}$ Unidad de Secuenciación/Unidad de diagnostico Molecular, Hospital Universitari Son Espases, Instituto de Investigación Sanitaria Illes Balears (IdISBa), Palma de Mallorca, Spain; ${ }^{3}$ Servicio de Traumatología, Hospital Universitari Son Espases, Instituto de Investigación Sanitaria Illes Balears (IdISBa), Palma de Mallorca, Spain; ${ }^{4}$ Servicio de Anatomía patológica, Hospital Universitari Son Espases, Instituto de Investigación Sanitaria Illes Balears (IdISBa), Palma de Mallorca, Spain

e-mail $\bowtie$ : manuel.diaz@ssib.es

Received: 28 March 2021; Accepted: 02 April 2021

Copyright: (C) 2021 Díaz-Cañestro M. This is an open-access article distributed under the terms of the Creative Commons Attribution License, which permits unrestricted use, distribution, and reproduction in any medium, provided that the original work is properly cited.

\section{ABSTRACT}

We present the case of a patient with right knee prosthetic arthritis due to Tropheryma whippelii in a 53-year-old man without other data of systemic involvement. The diagnosis was made by molecular microbiology techniques (bacterial identification by sequencing the $16 \mathrm{~S}$ rRNA). This case was managed with prosthetic replacement in two times and systemic antibiotic therapy.

Keywords: Prosthesis, Arthritis, Chronic, Knee, Tropheryma Whippelii

\section{Introduction}

Whipple's disease is a rare systemic infectious disorder that affects middle-aged white men and is caused by Tropheryma whipplei, a slow-growing facultative intracellular bacterium, an organism that is extremely difficult to culture. Morphologically this microorganism is sickle-shaped and stains with periodic Schiff acid (PAS), Gram and Giemsa stains (Fenollar et al., 2007).

The bacterium has been detected in sewage and is more frequent in faecal samples of sewage workers than the general population (Schoniger-Hekele et al., 2007). Importantly, not all the people who encounter the agent develop the disease (Schoniger-Hekele et al., 2007).

Classic Whipple's disease is a multisystemic process characterized by joint symptoms, chronic diarrhea, malabsorption and weight loss; although many other organ systems may also be affected. The disease occurs over time, with joint symptoms can precede others by many years, so not all symptoms can manifest at the time of presentation in affected people (Moreno Garcia et al., 2017; Meunier et al., 2013). The isolated involvement of other organs, mainly the central nervous system (CNS) and heart 
valves, can also occur in the absence of classical findings (Durand et al., 1997).

The standard for antibiotic therapy currently favors antibiotics that are capable of crossing the blood-brain barrier, such as trimethoprim-sulfamethoxazole. The recommended treatment is oral administration of $160 \mathrm{mg}$ of trimethoprim and $800 \mathrm{mg}$ of sulfamethoxazole twice per day for 1 to 2 years, usually preceded by parenteral administration of streptomycin (1 g per day) together with penicillin $\mathrm{G}$ (1.2 million U per day) or ceftriaxone (2 g daily) for 2 weeks (Fenollar et al., 2007).

Without treatment, Whipple's disease is ultimately fatal. Even with a specific antibiotic regimen, clinical relapse occurs in 2 to 33\% of cases after an average of 5 years; relapse is usually characterized by neurologic involvement (Fenollar et al., 2007).

Our aim is to present a patient with right knee prosthetic arthritis due to Tropheryma whippelii without other data of systemic involvement.

\section{Case Report}

A 53-year-old man, with a history of moderate enolism, without other pathological or epidemiological antecedents of interest, except for operated bilateral meniscectomia and later total right knee replacement in November 2016 due to bilateral gonarthrosis and subsequently mobilization under anesthesia (January 2017) and arthroscopic atrophy (April 2017) due to arthrofibrosis. He presented a torpid evolution with persistence of right gonalgia, local inflammatory signs and an increase in acute phase reactants with bone scintigraphy suggestive of septic loosening, with joint fluid bacteriological and fungal cultures were negative in November 2018.

In June 2019, the first time of the replacement is carried out in two stages with debridement and placement of dynamic cement spacer with antibiotic. Three intraoperative biopsies were performed that showed abundant leukocytes with negative bacteriological culture and PAS, PAS diastase and Grocott stains were negative. A genetic identification of the microorganism was requested by sequencing (rRNA 16s), being positive for Tropheryma whipple without referring the patient other joint involvement, history of valvulopathy, abdominal pain, picture diarrheal or constitutional. A study was completed with gastroscopy without macroscopic or histological alterations and transthoracic echocardiography without significant valvulopathy findings.

From the intervention, parenteral antibiotic treatment with vancomycin and ceftazidime was initiated for 2 weeks and after identification of the microorganism by sequencing, it was changed to oral cotrimoxazole and it was maintained for one more year with good clinical evolution and decreased 
inflammatory parameters

In October 2019, a second time of replacement was performed by removing the spacer and placing the Triathlon TS type PTR with tibial trabecular metal and allograft spongy chips. Intraoperative samples were obtained with culture and genetic identification of microorganisms by sequencing being negative (Fig. 1 and Fig. 2).

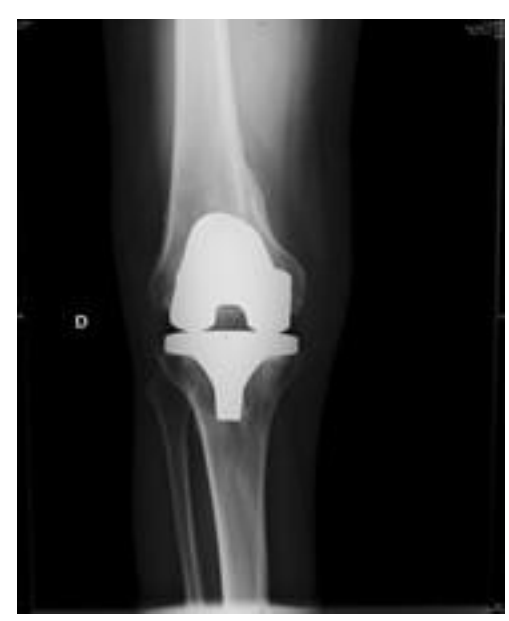

Figure 1: Knee prosthetic infection

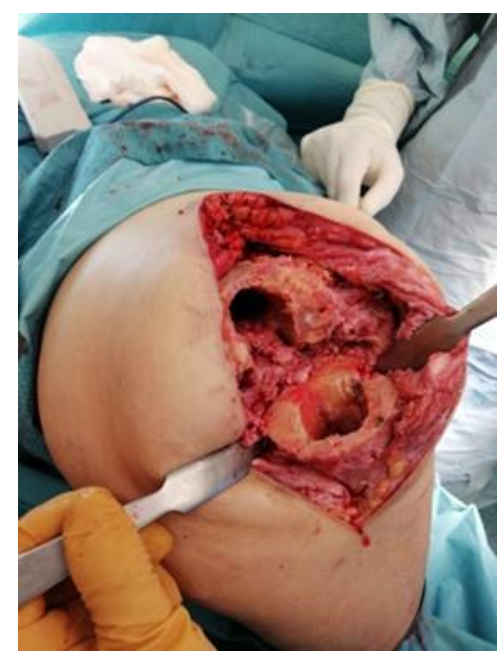

Figure 2: The second time of replacement: spacer removal and placement of Triathlon TS type PTR with tibial trabecular metal and allograft cancellous chips

\section{Discussion}

The relevance of our case lies on presenting itself as an apparently primary joint prosthetic infection without the systemic manifestations of classic Whipple's disease, which to our knowledge only two cases have been previously reported (Cremniter et al., 2008; Frésard et al., 1996). 
Whipple's disease is a rare systemic infectious process characterized by weight loss, arthralgia, diarrhea and abdominal pain, although it can have great clinical variability, affecting multiple organs, including the intestine, joints, central nervous system, eyes, heart, and lungs, being difficult to diagnose and controversial etiopathogenesis and treatment (Fenollar et al., 2007; Cremniter et al., 2008).

In reference to the pathogenesis of Whipple's disease, many people are exposed to T. whipplei, later the disease develops in some of these people with predisposing immune factors not yet defined (Fenollar et al., 2007). Genetic risk factors may be suggested by the predominance of males and the higher frequency of the HLA-B27 antigen among those with the disease. However, a causal association with any specific genetic factor has not been demonstrated (Fenollar et al., 2007). An impaired TH1 immune response is seen (Puechal, 2013). T whipplei replication in vitro is dependent on interleukin 16 and is accompanied by the apoptosis of host cells, facilitating dissemination of the bacterium (Puechal, 2013).

Rheumatologic manifestations are frequent, in $60 \%$ of cases, and precede digestive manifestations in three quarters of cases (Moreno Garcia et al., 2017). In many cases they constitute the first symptom of the disease (Fenollar et al., 2007; Moreno Garcia et al., 2017). It frequently manifests as polyarthritis, usually chronic, intermittent, seronegative and non-erosive, although cases of atypical rheumatologic manifestations such as spondylodiscitis, erosive arthritis, and even osteonecrosis of the hip have been described (Moreno Garcia et al., 2017; Meunier et al., 2013). The most commonly affected joints are the carpus and the large joints of the lower extremities (Moreno Garcia et al., 2017; Meunier et al., 2013).

In our case, the patient presented as a joint prosthetic infection without involvement of another joint, usually manifested as polyarthritis, or signs of systemic involvement.

Synovial and intestinal mucosal biopsy may show monocytic infiltrate stained with PAS (Fenollar et al., 2007). The development of the polymerase chain reaction (PCR) technique has allowed the identification of the genetic material of the bacteria in different tissues and body fluids, which suggests that the cause of arthritis in this disease is septic (Fenollar et al., 2007; Puechal, 2013). Initially, PCR assays targeting the 16S rRNA gene and the 16S-23S intergenic regions of the T. whippleigene were used (Fenollar et al., 2007). A new real-time quantitative PCR assay has recently been developed that targets repeated sequences of T. whipplei, with substantially greater sensitivity and the same specificity (Fenollar et al., 2007). It has also been possible to grow the bacteria from the synovial fluid (Fenollar et al., 2007; Meunier et al., 2013). Occasionally, joint study can be diagnostic, even if the bowel test is negative (Fenollar et al., 2007; Meunier et al., 2013).

In our case, the synovial biopsia, the bacteriological culture was negative, in line with the difficulty 
for growth in culture that this microorganism has shown (Fenollar et al., 2007), and PAS staining was negative. The diagnosis was obtained from the PCR, which has been described as the gold standard technique of Microbiological diagnosis, being more sensitive than PAS staining and the fastest and easiest way to diagnose Whipple's disease (Cremniter et al., 2008; Puechal, 2013). Therefore, we emphasize the importance of performing PCR in the proven cases of prosthetic joint infection with negative intraoperative cultures.

There is no clear consensus about treatment, with the use of intravenous ceftriaxone or another broad-spectrum beta-lactam being accepted for 2 weeks, followed by oral trimethoprim / sulfamethoxazole for a variable period, which is usually one year (Fenollar et al., 2007). A recent study recommends the combination of doxycycline and hydroxychloroquine for one year, subsequently maintaining doxycycline for life, to avoid relapses that occur with the previous regimen (Moos and Schneider, 2011). Clinical improvement is drastic, digestive earlier than articular, and neurological less predictable (Fenollar et al., 2007; Puechal, 2013).

The patient underwent a replacement of prostheses in two stages and was initiated intravenous antibiotics (ceftazidime) for two weeks and subsequently oral trimethoprim-sulfamethoxazole (Fenollar et al., 2007; Puechal, 2013) with good clinical evolution without the appearance of another systemic clinic.

\section{Conclusion}

In conclusion, primary infection of the joint prosthesis to Tropheryma whippelii is rare and even more so without the systemic manifestations of classic Whipple's disease. This report emphasizes the utility of applying universal PCR for the diagnosis and treatment of culture-negative prosthetic joint infections.

\section{References}

Cremniter J, Bauer T, Lortat-Jacob A, Vodovar D, Le Parc JM, Emile JF, Franc B, Sebbag P, Gaillard JL, Heym B. Prosthetic hip infection caused by Tropheryma whipplei.J Clin Microbiol 2008; 46: 1556-1557.

Durand DV, Lecomte C, Cathebras P, Rousset H, Godeau P. Whipple disease. Clinical review of 52 cases. The snfmi research group on whipple disease. Societe nationale francaise de medecine interne. Medicine (Baltimore) 1997; 76: 170-184.

Fenollar F, Puechal X, Raoult D. Whipple's disease. N Engl J Med 2007; 356: 55-66.

Frésard AG, Guglielminotti C, Berthelot P, Ros A, Farizon F, Dauga C, Rousset H, Lucht F. Prosthetic joint infection caused by tropheryma whippelii (whipple's bacillus). Clin Infect Dis 1996; 22: 575-576. 
Meunier M, Puechal X, Hoppé E, Soubrier M, Dieudé P, Berthelot JM, Caramaschi P, Gottenberg JE, Gossec L, Morel J, Maury E. Rheumatic and musculoskeletal features of whipple disease: A report of 29 cases. J Rheumatol 2013; 40: 2061-2066.

Moos V and Schneider T. Changing paradigms in Whipple's disease and infection with T. whipplei. Eur J Clin Microbiol Infect Dis 2011; 30: 1151-1158.

Moreno Garcia MS, Casorran Berges M, Del Rio-Martinez PS, Bosque Peralta MT. The great unknown, whipple's disease. Reumatol Clin 2017; 13: 243-244.

Puechal X. Whipple's disease. Ann Rheum Dis 2013; 72: 797-780.

Schoniger-Hekele M, Petermann D, Weber B, Muller C. Tropheryma whipplei in the environment: Survey of sewage plant influxes and sewage plant workers. Appl Environ Microbiol 2007; 73: 2033-2035. 